\title{
Predicting the Influence of Nano-scale Material Structure on the In-plane Buckling of Orthotropic Plates
}

\author{
Thomas S. Gates* \\ NASA Langley Research Center, Hampton, VA 23681 \\ Gregory M. Odegard ${ }^{\dagger}$ \\ National Institute of Aerospace, 144 Research Drive, Hampton, VA 23666 \\ Michael P. Nemeth \\ NASA Langley Research Center, Hampton, VA 23681 \\ Sarah-Jane V. Frankland ${ }^{\S}$ \\ National Institute of Aerospace, 144 Research Drive, Hampton, VA 23666
}

\begin{abstract}
A multi-scale analysis of the structural stability of a carbon nanotube-polymer composite material is developed. The influence of intrinsic molecular structure, such as nanotube length, volume fraction, orientation and chemical functionalization, is investigated by assessing the relative change in critical, in-plane buckling loads. The analysis method relies on elastic properties predicted using the hierarchical, constitutive equations developed from the equivalent-continuum modeling technique applied to the buckling analysis of an orthotropic plate. The results indicate that for the specific composite materials considered in this study, a composite with randomly orientated carbon nanotubes consistently provides the highest values of critical buckling load and that for low volume fraction composites, the nonfunctionalized nanotube material provides an increase in critical buckling stability with respect to the functionalized system.
\end{abstract}

\section{Introduction}

Development of high-stiffness and high-strength materials is an important part of the quest to advance aerospace vehicle structures. As a relatively new class of materials, single-walled carbon nanotube (SWNT) reinforced polymer composites provide many opportunities to demonstrate the performance potential of nanostructured materials for use in structural applications. In particular, SWNT materials have demonstrated the potential for order-of-magnitude increases in strength and stiffness relative to standard carbon-fibers used in typical polymeric composites. ${ }^{1}$

In contrast to carbon-fiber composites, the SWNT-polymer composites are considered nanostructured in the sense that the primary constituents have structure that can be readily described at the nano-scale. These material descriptions then provide the basis for the development of the underlying structure-property relationships that can be subsequently used in analysis models to tailor or enhance performance of structural components. To be truly useful, the structure-property relationships and related models must span multiple length scales and be versatile enough to be used in parametric studies that influence material development and design.

Reducing structural weight will continue to be one of the key design criteria for aerospace vehicles. Addressing this weight criterion while at the same time attempting to increase critical material properties, such as strength and stiffness, remains the primary challenge for the material developers. Eventually, in a robust design process, these enhancements to material properties must then translate into enhanced structural properties such as higher values of buckling resistance. It is this specific problem, the multi-scale linking of intrinsic, nano-scale material definitions to macro-scale structural stability that provides the motivation for this paper.

\footnotetext{
* Senior Materials Research Engineer, Mechanics and Durability, MS188E, Associate Fellow.

${ }^{\dagger}$ Staff Scientist, Senior Member.

* Senior Research Engineer, Mechanics and Durability, MS188E, Associate Fellow.

${ }^{\S}$ Staff Scientist.
} 
Buckling resistance is an important consideration in the design of thin-walled, lightweight aerospace vehicle structures. Thus, studies of the effects of SWNT structure on the global buckling resistance of a basic structural element, such as a flat plate, serve as a first-order means for assessing the potential of SWNT-reinforced polymeric composites. As a natural progression toward this type of global assessment, several studies that examine the compressive buckling behavior of an individual nanotube, unsupported by a surrounding matrix, have been conducted using a range of analysis techniques. The typical representation of a SWNT is a cylindrical, lattice structure of carbon atoms with a tube diameter of approximately one nanometer and tube length varying from tens of nanometers to ten of microns. To investigate the influence of van der Waals forces on the compressive buckling of double-walled nanotubes, $\mathrm{Ru}^{2}$ developed a model based on an elastic shell analysis. In a related study, Pantano, et al. ${ }^{3}$ utilized shell finite elements with the van der Waals interactions modeled with special interaction elements. Both compressive buckling and post-buckling behavior were predicted and compared with molecular dynamics results.

The more general problem of structural stability of heterogeneous materials and the influence of scale has also been examined recently by Miehe, et $\mathrm{al}^{4}{ }^{4}$ Extending down to the microscale, this study examined periodic, finescale material structures using a scaled continuum approach. This coupled, macro-to-micro approach revealed that one of the primary modeling obstacles was the selection of the relevant size of the representative volume element. Using a strictly continuum approach, Parnes, et al. ${ }^{5}$ modeled the influence of reinforcement volume fraction on inplane buckling of a composite plate. In Parnes study, it was assumed that carbon nanotube reinforced polymers would behave in a similar manner to the predicted behavior of dilute or low volume fraction composites.

The emphasis of these previous studies was on selected modes of failure associated with compression loading: nanotube buckling and local stability. The objective of the present paper is to develop a multi-scale analysis model to predict the influence of molecular attributes on the macro-scale, global structural stability of an SWNTpolymer composite plate subjected to a general state of in-plane mechanical loads. The determination of the SWNTpolymer composite elastic stiffness properties used in the current study was described previously by Odegard, et al. ${ }^{6}$ In this previous study, the mechanical behavior of the SWNT-polymer composite was assumed to depend not only on the individual properties of the polymer and the nanotubes, but also on the nanotube/polymer interaction. Four molecular-level structural items were defined by this previous study as the primary molecular attributes, controlled at material synthesis, which could influence final properties of the composite. These four attributes were nanotube length, volume fraction, nanotube orientation, and nanotube functionalization. The latter item, nanotube functionalization, was defined as the formation of a chemical covalent bond between the nanotube and polymer directly. The influence of these four intrinsic structure attributes on bulk constitutive properties was investigated using a hierarchical modeling approach.,

To accomplish the objective of the present study, results are presented herein that illustrate the effects of nanotube functionalization, nanotube length, volume fraction, and nanotube orientation on the elastic stiffness properties and buckling resistance of a single-walled carbon-nanotube-reinforced polymeric-composite plate. First, the equivalent-continuum modeling approach and the micromechanical analysis are used to determine the elastic material properties of the composite are described. Next, nondimensional parameters and equations that define the buckling behavior of thin, specially orthotropic plates that are subjected to uniform axial compression, shear, or pure in-plane bending are presented. More specifically, equations for infinitely long plates with simply supported or with clamped edges are presented. Finally, results are presented and discussed that illustrate the effects of nano-scale structural parameters on the buckling resistance of the composite plates.

\section{Materials}

The material system used in this study was a carbon nanotube reinforced polymer composite. The carbon nanotube material was modeled as a single-walled $(10,10)$ nanotube of radius $6.78 \AA$ and variable length. The polymer matrix material was assumed to be isotropic, amorphous polyethylene matrix with a representative Young's modulus and Poisson's ratio of $0.9 \mathrm{GPa}$ and 0.3 , respectively.

\section{Equivalent-Continuum Modeling}

Using a hierarchical modeling scheme, the equivalent-continuum modeling technique of Odegard, et al. ${ }^{7,8}$ was used to predict the bulk elastic properties. Briefly, the hierarchical modeling method relies on three major steps. First, a suitable representative volume element (RVE) of the nano-structured material is chosen based on the geometry of the molecular model. Second, an equivalent-truss model of the RVE is developed as an intermediate step to link the molecular and equivalent-continuum models. Finally, an equivalent-continuum model of the RVE is developed in which the total strain energy in the molecular and equivalent-continuum models, under identical

loading conditions, is equal. The effective mechanical properties of the equivalent-continuum are then determined directly by equating strain energies of the systems. 


\section{A. Molecular Model}

The RVE of a typical nano-structured material is on the nanometer length scale, therefore, the material of the $\mathrm{RVE}$ is not continuous, but is an assemblage of many atoms. Interaction of these atoms is described with a molecular-mechanics force field. ${ }^{9,10}$ The molecular model represents the RVE of the equilibrium molecular structure of the nano-structured material.

Molecular dynamics (MD) simulations were used in the present study to determine the equilibrium structures of the nonfunctionalized and functionalized composite. For the two composite systems, the starting configuration for the MD simulation was a crystalline polyethylene matrix containing a $(10,10)$ single-walled carbon nanotube. For both models, the nanotube and polymer chains were replicated across the periodic boundaries of the simulation cell, making them infinitely long. In the functionalized composite, two polymer chains were attached to six carbon atoms of the nanotube by chemical linkages consisting of $2 \mathrm{CH}_{2}$ groups. Equilibration of these initial structures was carried out with molecular dynamics simulation using the Brenner potential to describe all the chemical bonds in the system. The van der Waals interactions were simulated with a Lennard-Jones potential. The resulting structures were then used to compose the RVE. In the RVE, the molecular mechanics force field defined the interactions of the atoms in terms of bond stretching, bond-angle variation, and Van der Waals (Lennard-Jones) interactions. ${ }^{8}$

\section{B. Truss Model}

The geometry of the molecular structure defined by the MD simulations was used to define an equivalenttruss structure. To implement the resultant equivalent-truss structures, finite element models were developed, where each element was a three-dimensional pin-jointed truss element with six degrees of freedom (three displacement components on each end) that represented a single atomic interaction. Each node corresponded to an atom in the equilibrium structure of the molecular model. The Young's moduli of the truss elements were determined such that the total molecular potential energy of the molecular model and the strain energy of the equivalent-truss are equal for the same loading conditions.

\section{Continuum Model}

With the equivalent-truss structure defined, the continuum models were constructed. The geometries of the homogeneous, equivalent-continuum RVE's were assumed to be cylindrical, similar to that of the molecular and truss models. The mechanical properties of the solid-cylinder continuum models were determined by equating the total strain energies of the equivalent-truss and equivalent-continuum models under identical loading conditions. The molecular model of the functionalized nanotube/polyethylene composite was assumed to exhibit the symmetry of an orthotropic material. Therefore, only nine independent material parameters were required to determine the entire set of elastic constants. Each of the nine parameters was determined from a single boundary condition that was applied to both the equivalent-truss and equivalent-continuum models. Once the mechanical properties of the equivalent-continuum RVE's were determined, the two composite material RVE's were assumed to behave in the composite as effective fibers and were used in subsequent micromechanical analyses to predict the influence of effective fiber volume fraction, orientation, and length.

\section{Effective-Fiber Constitutive Model}

The constitutive relationship of an orthotropic equivalent-continuum RVE (which is henceforth also referred to as an effective fiber) is

$$
\begin{gathered}
\sigma_{11}=C_{11}^{f} \varepsilon_{11}+C_{12}^{f} \varepsilon_{22}+C_{13}^{f} \varepsilon_{33} \\
\sigma_{22}=C_{12}^{f} \varepsilon_{11}+C_{22}^{f} \varepsilon_{22}+C_{23}^{f} \varepsilon_{33} \\
\sigma_{33}=C_{13}^{f} \varepsilon_{11}+C_{23}^{f} \varepsilon_{22}+C_{33}^{f} \varepsilon_{33} \\
\sigma_{23}=2 C_{44}^{f} \varepsilon_{23} \\
\sigma_{13}=2 C_{55}^{f} \varepsilon_{13} \\
\sigma_{12}=2 C_{66}^{f} \varepsilon_{12}
\end{gathered}
$$

where $\sigma_{i j}$ and $\varepsilon_{i j}$ are the stress and strain components, respectively $(i, j=1,2,3)$, and $C_{k l}^{f}$ are the elastic stiffnesses of the effective fiber (denoted by superscript $f$ ) which are written in Voigt's abridged index notation $(k, l=1, \ldots, 6)$. 
Nine independent elastic properties are required to describe the overall elastic behavior of an orthotropic material, as mentioned previously and shown in Eq. (1). For convenience, the nine independent elastic properties that are used to describe the overall behavior of the effective fiber are the three elastic axial stiffnesses, $C_{11}^{f}, C_{22}^{f}$, and $C_{33}^{f}$; the three plane-strain bulk moduli, $K_{23}^{f}, K_{13}^{f}$, and $K_{12}^{f}$; and the three elastic shear stiffnesses, $C_{44}^{f}, C_{55}^{f}$, and $C_{66}^{f}$. The three plane-strain bulk moduli are defined as

$$
\begin{aligned}
& K_{23}^{f}=\frac{1}{4}\left(C_{22}^{f}+C_{33}^{f}+2 C_{23}^{f}\right) \\
& K_{13}^{f}=\frac{1}{4}\left(C_{11}^{f}+C_{33}^{f}+2 C_{13}^{f}\right) \\
& K_{12}^{f}=\frac{1}{4}\left(C_{11}^{f}+C_{22}^{f}+2 C_{12}^{f}\right)
\end{aligned}
$$

where the subscripts indicate the plane that is subjected to a plane-strain deformation. Once the nine independent elastic properties are determined, the elastic interaction stiffness components, $C_{23}, C_{13}$, and $C_{12}$, can be calculated from the relations in Eq. (2).

At this point in the model development, the values of the nine elastic parameters are unknown. These values are determined by applying nine identical sets of boundary conditions to the equivalent-truss model and the effective fiber, and by subsequently equating the strain energies by adjusting the nine independent elastic properties. The calculated values of the nine independent parameters for the functionalized and nonfunctionalized effective fibers are listed in Table 1.

Table 1. Equivalent-Continuum Properties (GPa).

\begin{tabular}{|c|c|c|}
\hline & $\begin{array}{c}\text { Nonfunctionalized } \\
\text { effective fiber }\end{array}$ & $\begin{array}{c}\text { Functionalized } \\
\text { effective fiber }\end{array}$ \\
\hline$C_{11}^{f}$ & 548.7 & 487.7 \\
\hline$C_{22}^{f}$ & 16.8 & 24.5 \\
\hline$C_{33}^{f}$ & 16.5 & 20.6 \\
\hline$K_{23}^{f}$ & 14.8 & 19.5 \\
\hline$K_{13}^{f}$ & 149.3 & 137.1 \\
\hline$K_{12}^{f}$ & 149.2 & 138.7 \\
\hline$C_{44}^{f}$ & 7.1 & 12.7 \\
\hline$C_{55}^{f}$ & 144.0 & 155.4 \\
\hline$C_{66}^{f}$ & 144.9 & 137.0 \\
\hline
\end{tabular}

\section{Micromechanical Modeling}

Constitutive models of the effective fiber/polymer composites were obtained from a micromechanical analysis by using the mechanical properties of the nanotube/polymer effective fibers and the bulk polymer matrix material. For the composites considered in this study, the polymer molecules that were near the polymer/nanotube interface were included in the effective fiber, and it was assumed that the polymer matrix surrounding the effective fiber had mechanical properties equal to those of bulk polyethylene. It was also assumed that perfect bonding existed between the nanotube/polymer effective fibers and the surrounding polymer matrix in the micromechanics analysis. 
A micromechanics-based Mori-Tanaka method was used to predict the elastic mechanical properties of the composite material. ${ }^{11}$ For this method, the overall elastic-stiffness tensor of the composite containing orthotropic effective fibers embedded in a matrix material is

$$
\mathbf{C}=\left(c_{m} \mathbf{C}_{m}+c_{f}\left\langle\mathbf{C}_{f} \mathbf{T}_{f}\right\rangle\right)\left(c_{m} \mathbf{I}+c_{f}\left\langle\mathbf{T}_{f}\right\rangle\right)^{-1}
$$

where $c_{f}$ and $c_{m}$ are the effective fiber and matrix volume fractions, respectively, $\mathbf{C}_{f}$ and $\mathbf{C}_{m}$ are the stiffness tensors of the effective fiber and matrix, respectively, $\mathbf{I}$ is the identity tensor, the angle brackets indicate an effective-fiber orientation average, and $\mathbf{T}_{f}$ is the dilute strain-concentration tensor of the effective fiber, and is given by

$$
\mathbf{T}_{f}=\left[\mathbf{I}+\mathbf{S C}_{m}^{-1}\left(\mathbf{C}_{f}-\mathbf{C}_{m}\right)\right]^{-1}
$$

where $\mathbf{S}$ is the Eshelby ${ }^{12}$ tensor, which is given in detail elsewhere. ${ }^{13}$

While it is convenient to establish constitutive equations for composites in terms of the homogenizedcomposite stiffness tensor, $\mathbf{C}$, The Young's and shear moduli are the elastic constants that are most often used to compare mechanical properties. While the shear moduli of the composite material $\left(G_{44}, G_{55}, G_{66}\right)$ are simply equal to the shear-stiffnesses $\left(C_{44}, C_{55}, C_{66}\right)$, the Young's moduli $\left(E_{1}, E_{2}, E_{3}\right)$ were calculated by using the components of the compliance tensor of the composite material, which was determined by inverting the composite stiffness tensor, $\mathbf{C}$ of the composite. ${ }^{14}$ The subscripts of the Young's moduli and shear moduli indicate the principal direction associated with the quantity, similar to Eq. (1). Therefore, $E_{1}$ is the longitudinal Young's modulus (parallel to the nanotube), $E_{2}$ and $E_{3}$ are transverse Young's moduli, $G_{44}$ is the transverse shear modulus, and $G_{55}$ and $G_{66}$ are longitudinal shear moduli. In a random composite, defined as a material with randomly oriented fibers distributed in a uniform manner, the material is assumed to be isotropic. For the isotropic, random composite, mechanical properties are completely described by the Young's modulus, $E\left(E=E_{1}=E_{2}=E_{3}\right)$, and shear modulus, $G\left(G=G_{44}=\right.$ $\left.G_{55}=G_{66}\right)$.

For the effective fiber/polymer composites considered in the present study, the elastic stiffness components, volume fraction, length, and orientation of the effective fiber were used for the fiber properties in Eq. (3). The calculations were performed for both perfectly aligned and three-dimensional randomly oriented effective fibers.

\section{Plate Buckling Equations}

The plate buckling equations used to assess the benefits of the SWNT-reinforced polymeric composites considered in the present study are the simple closed-form equations presented by Nemeth for specially orthotropic plates. ${ }^{15,16}$ These equations are for infinitely long plates with a constant thickness $(t)$, a constant width $(b)$, and with either simply supported or clamped edges. The solutions for infinitely long plates are particularly useful in preliminary design of structural elements because they represent lower bounds to the corresponding festoon buckling-load-versus-plate-aspect-ratio curves for finite-length plates. Moreover, the equations are based on classical plate theory and are for plates that are subjected to uniform axial compression $\left(N_{X}\right)$, uniform shear $\left(N_{S}\right)$, or pure in-plane bending loads $\left(N_{B}\right)$, as shown in Fig. 1. As also shown in Fig. 1, the plate coordinates are designated $(x, y)$ and the material coordinates are designated $(1,2,3)$. For the composites considered herein, both coordinate systems are coincident. Moreover, the plate is presumed to consist of a single layer of homogeneous, specially orthotropic material.

Classical plate theory assumes that each layer of material is in a state of plane stress. For the single layer of homogenized material considered for the plates herein, the material constitutive relation is given in terms of the nonzero principal-material-direction stresses by

$$
\left\{\begin{array}{l}
\sigma_{11} \\
\sigma_{22} \\
\sigma_{12}
\end{array}\right\}=[Q]\left\{\begin{array}{l}
\varepsilon_{11} \\
\varepsilon_{22} \\
\gamma_{12}
\end{array}\right\}
$$

where the stiffness matrix, commonly called the reduced stiffness matrix, [Q] is given explicitly by 


$$
[Q]=\left[\begin{array}{ccc}
\frac{E_{1}}{1-v_{12} \nu_{21}} & \frac{v_{12} E_{2}}{1-v_{12} \nu_{21}} & 0 \\
\frac{v_{12} E_{2}}{1-v_{12} \nu_{21}} & \frac{E_{2}}{1-v_{12} \nu_{21}} & 0 \\
0 & 0 & G_{12}
\end{array}\right]
$$

and the four independent elastic constants are $\mathrm{E}_{1}, \mathrm{E}_{2}, \mathrm{G}_{12}$, and $v_{12}$. For a single-layer orthotropic plate, the bending stiffness matrix [D] is then given by

$$
[D]=\frac{t^{3}}{12}[Q]
$$

As is well known, the buckling behavior of thin plates is represented by a fourth-order partial differential equation, with homogeneous boundary conditions. The critical, or buckling, load is found by solving the associated boundary-eigenvalue problem for the smallest value of the applied load. For the three distinct types of in-plane loads considered herein (compression, shear, and bending), the buckling formulas are given in terms of the nondimensional buckling coefficients given by

$$
\begin{aligned}
& K_{X}=\frac{N_{X}^{c r} b^{2}}{\pi^{2} \sqrt{D_{11} D_{22}}} \\
& K_{S}=\frac{N_{S}^{c r} b^{2}}{\pi^{2} \sqrt[4]{D_{11} D_{22}^{3}}} \\
& K_{B}=\frac{N_{B}^{c r} b^{2}}{\pi^{2} \sqrt{D_{11} D_{22}}}
\end{aligned}
$$

where the superscript $c r$ denotes the critical value of the applied load that corresponds to buckling. The simplified buckling equations are presented in table 2 and were determined by performing a least-squares fit to response curves determined by a special-purpose Rayleigh-Ritz analysis. The buckling coefficients given in table 2 depend only on a single nondimensional parameter that is given by

$$
\beta=\frac{D_{12}+2 D_{66}}{\sqrt{D_{11} D_{22}}}
$$

Nemeth provides the following simplified linear expressions for the buckling coefficient:

Table 2. Buckling coefficients for in-plane loading.

\begin{tabular}{|c|c|c|}
\hline & Simply supported edges & Clamped edges \\
\hline$K_{X}$ & $2+2 \beta$ & $4.602+2.359 \beta$ \\
\hline$K_{S}$ & $3.629+1.644 \beta$ & $6.493+2.414 \beta$ \\
\hline$K_{B}$ & $13.425+10.449 \beta$ & $26.947+12.667 \beta$ \\
\hline
\end{tabular}

To make direct comparisons of plate buckling resistance, the buckling coefficients defined herein are not the best choice. It is more useful and convenient to use a buckling load that is nondimensionalized with respect to the bending stiffness of an isotropic plate made of the bulk polymer material. For the bulk polymer matrix material, let $E_{p}$ and $v_{p}$ be defined as the Young's modulus and Poisson's ratio respectively. The bending stiffness for an isotropic, bulk polymer plate is then given as 


$$
\bar{D}=\frac{E_{p} t^{3}}{12\left(1-v_{p}^{2}\right)}
$$

To obtain the desired nondimensional buckling loads, it is convenient to define the nondimensional stiffness ratio

$$
D^{*}=\frac{\sqrt{D_{11} D_{22}}}{\bar{D}}
$$

giving the following definitions of the nondimensional buckling loads.

$$
\begin{array}{ll}
N_{X}^{*}=K_{X} D^{*} & \text { compression } \\
N_{S}^{*}=K_{S} D^{*} & \text { shear } \\
N_{B}^{*}=K_{B} D^{*} & \text { bending }
\end{array}
$$

\section{Results and Discussion}

In this section, the elastic constants and critical buckling behavior of the three SWNT-polymer composite are presented as a function of nanotube length, volume fraction, orientation, and functionalization. Based on these results, implications for design and material development are discussed.

\section{A. Elastic Properties of Composites}

The longitudinal and transverse Young's moduli and the shear modulus of the homogenized, nanotubereinforced composites are plotted in Figs. 2 and 3 respectively, as a function of nanotube length, for a $1 \%$ nanotube volume fraction. Two groups of curves are shown in the figures that correspond to composites with the nanotubes either longitudinally or transversely aligned, like well-known unidirectional carbon-fiber-reinforced composites. A third group of curves is shown in the figures for the case in which the nanotubes are randomly oriented, like chopped-fiber composites that are commonly found in practice.

The data in Figs. 2 and 3 indicate that at a nanotube length of about $400 \mathrm{~nm}$, the efficiency of load transfer between the SWNT and the matrix material is nearly maximized. At a length of $450 \mathrm{~nm}$, functionalization reduces the longitudinal Young's modulus $\left(E_{l}\right)$ of the aligned composite and the Young's modulus of the random composite by $11 \%$ and $7 \%$, respectively. In contrast, the transverse Young's modulus of the aligned composite exhibits no dependence on nanotube length or functionalization. Based on the results presented in Fig. 3, functionalization decreased the shear modulus of the random composites whereas there was no significant effect of functionalization on the shear modulus of the aligned composite as the nanotube length was increased.

The longitudinal and transverse Young's moduli of the random and aligned composites are shown in Figs. 4 and 5 respectively, as a function of nanotube volume fraction, for a constant nanotube length of $400 \mathrm{~nm}$. Two groups of curves are shown in Fig. 4 that corresponds to composites with the nanotubes either longitudinally or randomly aligned. A single group of curves is shown in Fig. 5 that corresponds to transverse alignment of the nanotubes. Over the complete range of nanotube volume fraction shown, the functionalization of the nanotube reduced the longitudinal Young's modulus of the composite. In contrast, the transverse Young's moduli of the composite improved when the nanotubes were functionalized. The enhancement is evident for nanotube volume fractions greater than $10 \%$.

\section{B. Buckling Behavior}

Nondimensional buckling loads for all three loading conditions and both boundary conditions (Table 2) previously described were calculated as a function of nanotube length, volume fraction, orientation, and functionalization. These predicted results are plotted in Figs. 6-17.

The first set of buckling results, presented in Figs. 6-11, represent the nondimensional buckling loads as a function of nanotube length for a fixed $1 \%$ volume fraction. For all cases, including all loading and support conditions, the nondimensional buckling load was found to be a strong function of nanotube length with an increase in nanotube length resulting in a corresponding increase in buckling load. The rate of change varies according to alignment and functionalization, but in particular, the rate of change in buckling was most significant at nanotube lengths below $200 \mathrm{~nm}$. For the compression and bending load cases, a divergence between the buckling load of the functionalized and nonfunctionalized composites occurred as nanotube length exceeded the $200 \mathrm{~nm}$ range. In general, for all loading cases, the nondimensional buckling loads for a clamped boundary condition exceeded the 
simply supported cases. For nanotubes lengths greater than $200 \mathrm{~nm}$, the highest buckling loads are exhibited by the randomly aligned, nonfunctionalized composite.

The second set of buckling results, presented in Figs. 12-17, represent the nondimensional buckling loads as a function of nanotube volume fraction, for a fixed nanotube length of $400 \mathrm{~nm}$. In general, for all cases (including all loading and support conditions), the composites reinforced with randomly oriented nanotubes exhibit upwards of a six-fold increase in buckling resistance with nanotube volume fraction, as compared to the composites reinforced with axially aligned nanotubes. The differences between the results predicted for the composites reinforced with randomly oriented and axially aligned nanotubes are the most apparent for nanotube volume fractions above $10 \%$. Another general observation is that the trends exhibited by the buckling load versus volume fraction curves are not a function of loading or boundary conditions. Once again, the highest buckling resistance occurs for the nonfunctionalized composites reinforced with randomly aligned nanotubes.

\section{Summary and Conclusions}

The macroscopic buckling resistance of composite plates made of a polyethylene matrix material that is reinforced with single-walled carbon nanotubes has been presented. In particular, the relative influence of molecular structure, nanotube length, and nanotube volume fraction on buckling resistance of infinitely long plates subjected to uniform axial compression, uniform shear, or pure in-plane bending have been quantified for both functionalized and nonfunctionalized nanotubes. A multi-scale analysis method was developed that established constitutive equations by using a hierarchical equivalent-continuum modeling technique that predicted elastic bulk behavior, using intrinsic properties developed through molecular dynamics simulations. The elastic properties of the equivalent continuum were then used in a micromechanics analysis to predict elastic properties of a single-layer specially orthotropic plate as a function of nanotube alignment. The predictions of buckling resistance were based on these resultant elastic properties.

The results presented herein show that for a fixed nanotube volume fraction of $1 \%$ and various nanotube lengths, the Young's and shear moduli of the composites reinforced with randomly oriented fibers and the longitudinal Young's moduli of composites with aligned fibers have been shown to decrease up to $11 \%$ when the nanotube is functionalized. In general, the buckling resistance of the random, nonfunctionalized nanotube composite exceeded all other cases with the increase in nondimensional buckling load ranging up to 25\% (at nanotube length of $400 \mathrm{~nm}$ ) when compared to the composites reinforced with the aligned, functionalized nanotubes.

The results that have been presented herein also show that for a fixed nanotube length of $400 \mathrm{~nm}$ and various nanotube volume fractions, the longitudinal Young's moduli of the composites reinforced with the aligned nanotubes and both the Young's and shear moduli of the composite with the randomly oriented fibers have also been shown to decrease up to $12 \%$ when the nanotube is functionalized. However, under these conditions, the transverse Young's moduli and the transverse shear moduli of the aligned composites have shown an increase of up to 45\% (at $40 \%$ volume fraction) when the nanotube is functionalized. The buckling resistance of the random nanotube composites exceeded all other cases with the increase in buckling resistance ranging up to $500 \%$ (at $40 \%$ volume fraction) when compared to the aligned composite. At the large volume fractions, the influence of functionalization was to slightly decrease critical buckling loads.

Based on all the cases considered in the present study, it appears that the use of randomly oriented, nonfunctionalized nanotubes would be recommended when the design criteria is focused on providing the largest possible value of in-plane buckling resistance. The primary elastic properties that influence this result are the composite-plate stiffnesses that correspond to twisting and anticlastic curvature. To achieve the largest gains in buckling resistance, nanotube volume fractions greater than $20 \%$ are warranted.

\section{References}

1. Thostenson, E.T., Z. Ren, and T.W. Chou, Advances in the Science and Technology of Carbon Nanotubes and Their Composites: A Review, Composites Science and Technology, 2001, 61: pp. 1899-1912.

2. Ru, C.G., Effect of van der Waals forces on axial buckling of a double-walled carbon nanotube, Journal of Applied Physics, 2000, 87(10): pp. 7227-7231.

3. Pantano, A., M.C. Boyce, and D.M. Parks, Nonlinear Structural Mechanics Based Modeling of Carbon Nanotube Deformation, Physical Review Letters, 2003, 91(14): pp. 145504-1 145504-4.

4. Miehe, C., J. Schroder, and M. Becker, Computational homogenization analysis in finite elasticity: material and structural instabilities on the micro- and macro-scales of periodic composites and their interaction, Computer Methods in Applied Mechanics and Engineering, 2002, 191: pp. 4971-5005. 
5. Parnes, R. and A. Chiskis, Buckling of nano-fibre reinforced composites: a re-examination of elastic buckling, Journal of the Mechanics of Physics and Solids, 2002, 50: pp. 855-879.

6. Odegard, G.M., S.J.V. Frankland, T.S. Gates, The Effect of Chemical Functionalization on Mechanical Properties of Nanotube/Polymer Composites in 44th AIAA/ASME/ASCE/AHS/ASC SDM Conference, April 7-10, 2003, Norfolk, VA, AIAA-2003-1701.

7. Odegard, G.M., T.S. Gates, L.M. Nicholson, and K.E. Wise, Equivalent-Continuum Modeling of Nano-Structured Materials, Journal of Composites Science and Technology, 2002, 62(14): pp. 1869-1880.

8. Odegard, G.M., et al., Constitutive Modeling of Nanotube-Reinforced Polymer Composites, Journal of Composites Science and Technology: Modeling and Characterization of Nanostructured Materials, 2003, 63(11): pp. 1671-1687.

9. Rappe, A.K. and C.J. Casewit, Molecular Mechanics Across Chemistry, 1997, Sausalito: University Science Books.

10. W. D. Cornell, P.C., C. I. Bayly, I. R. Gould, K. M. Merz, D. M. Ferguson, D. C. Spellmeyer, T. Fox, J. W. Caldwell, P. A. Kollman, A Second Generation Force Field for the Simulation of Proteins, Nucleic Acids, and Organic Molecules, Journal of the American Chemical Society, 1995, 117: pp. 5179-5197.

11. Mori, T. and K. Tanaka, Average Stress is Matrix and Average Elastic Energy of Materials with Misfitting Inclusions, Acta Metallurgica, 1973, 21: pp. 571-574.

12. Eshelby, J.D., The Determination of the Elastic Field of an Ellipsoidal Inclusion, and Related Problems in 1957, Series A, 241: Proceedings of the Royal Society of London, pp.376-396.

13. Mura, T., Micromechanics of Defects in Solids, 1982, The Hague: Martinus Nijhoff.

14. Hull, D. and T.W. Clyne, An Introduction to Composite Materials, 1996, Cambridge: Cambridge University Press.

15. Nemeth, M.P., Buckling of Long Compression-Loaded Anisotropic Plates Restrained Against Inplane Lateral and Shear Deformation in 44th Annual AIAA/ASME/ASCE/AHS/ASC Structures, Structural Dynamics, and Materials Conference, April 7-10, 2003, Norfolk, VA, AIAA-2003-1789, pp.37.

16. Nemeth, M.P., Buckling Behavior of Long Anisotropic Plates Subjected to Restrained Thermal Expansion and Mechanical Loads, Journal of Thermal Stresses, 2000, 23: pp. 873-916.

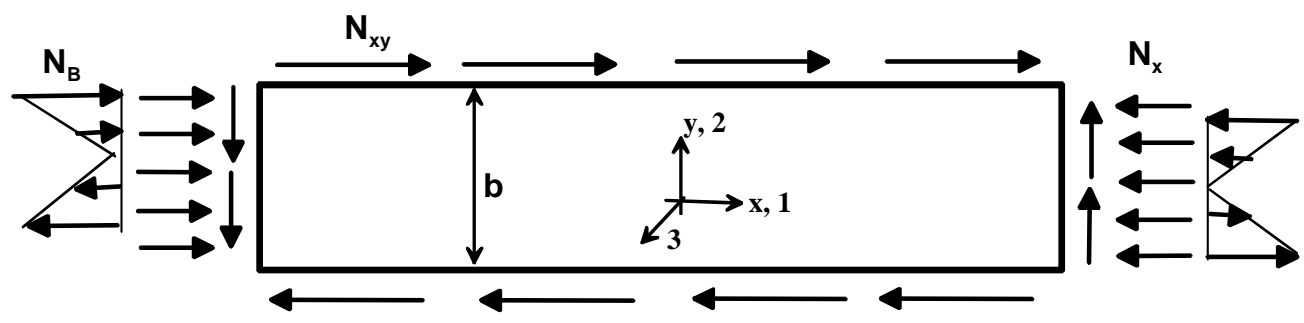

Figure 1. Schematic illustration of the mechanical loads and coordinate directions for the infinitely long, orthotropic plate. 


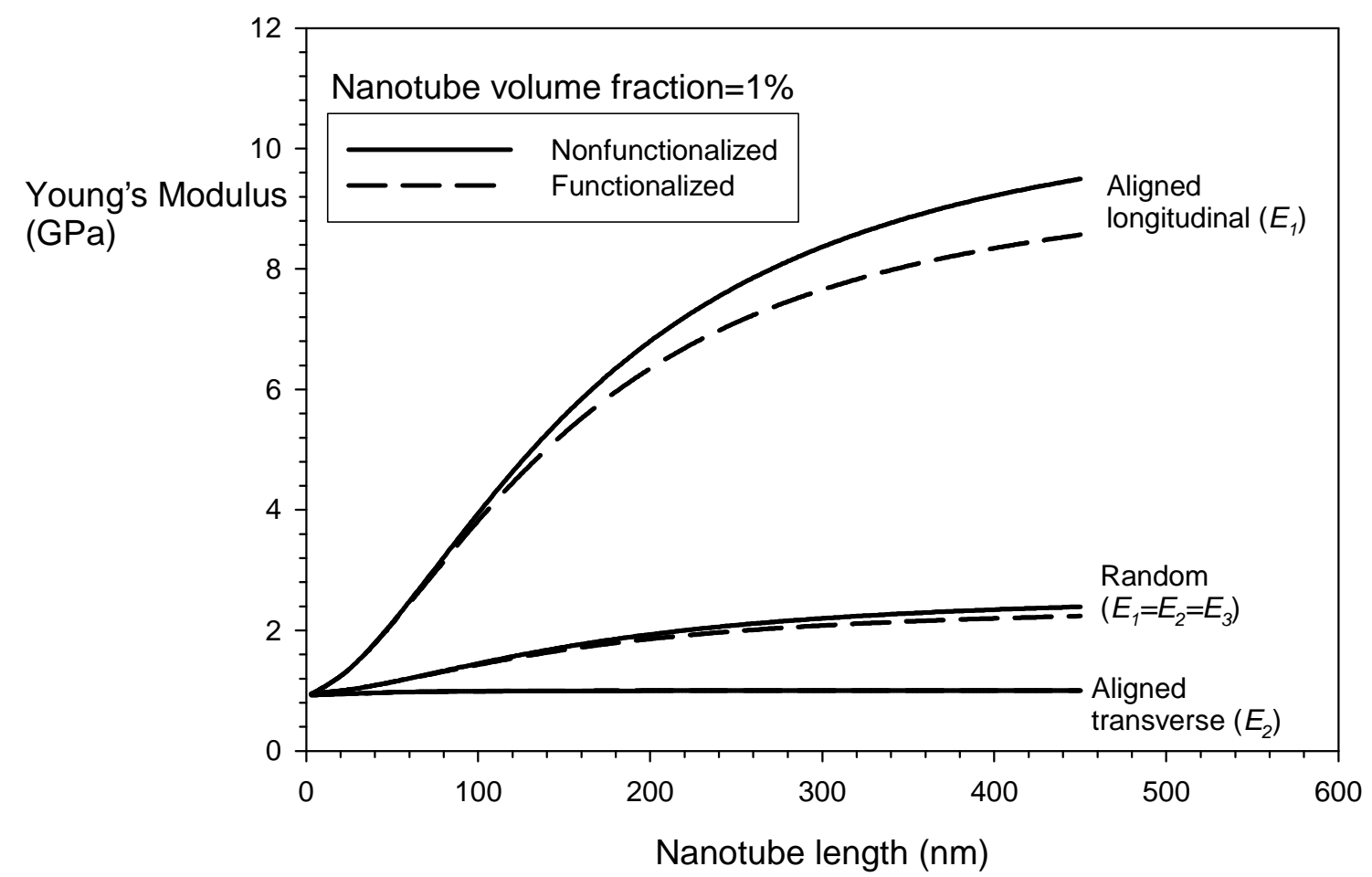

Figure 2: Effect of nanotube length on longitudinal and transverse Young's modulus, for fixed volume fraction.

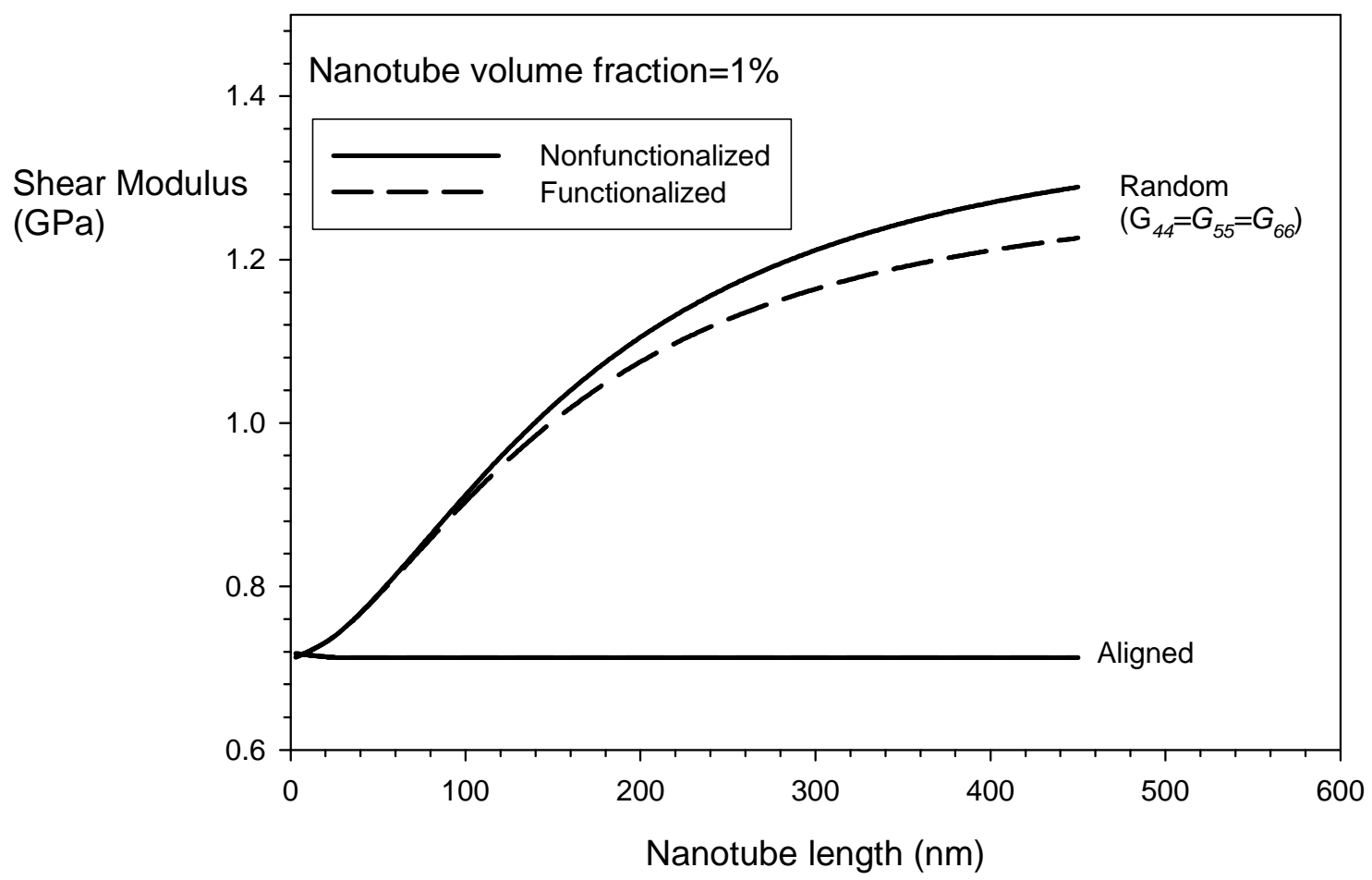

Figure 3: Effect of nanotube length on e shear modulus, for fixed volume fraction. 


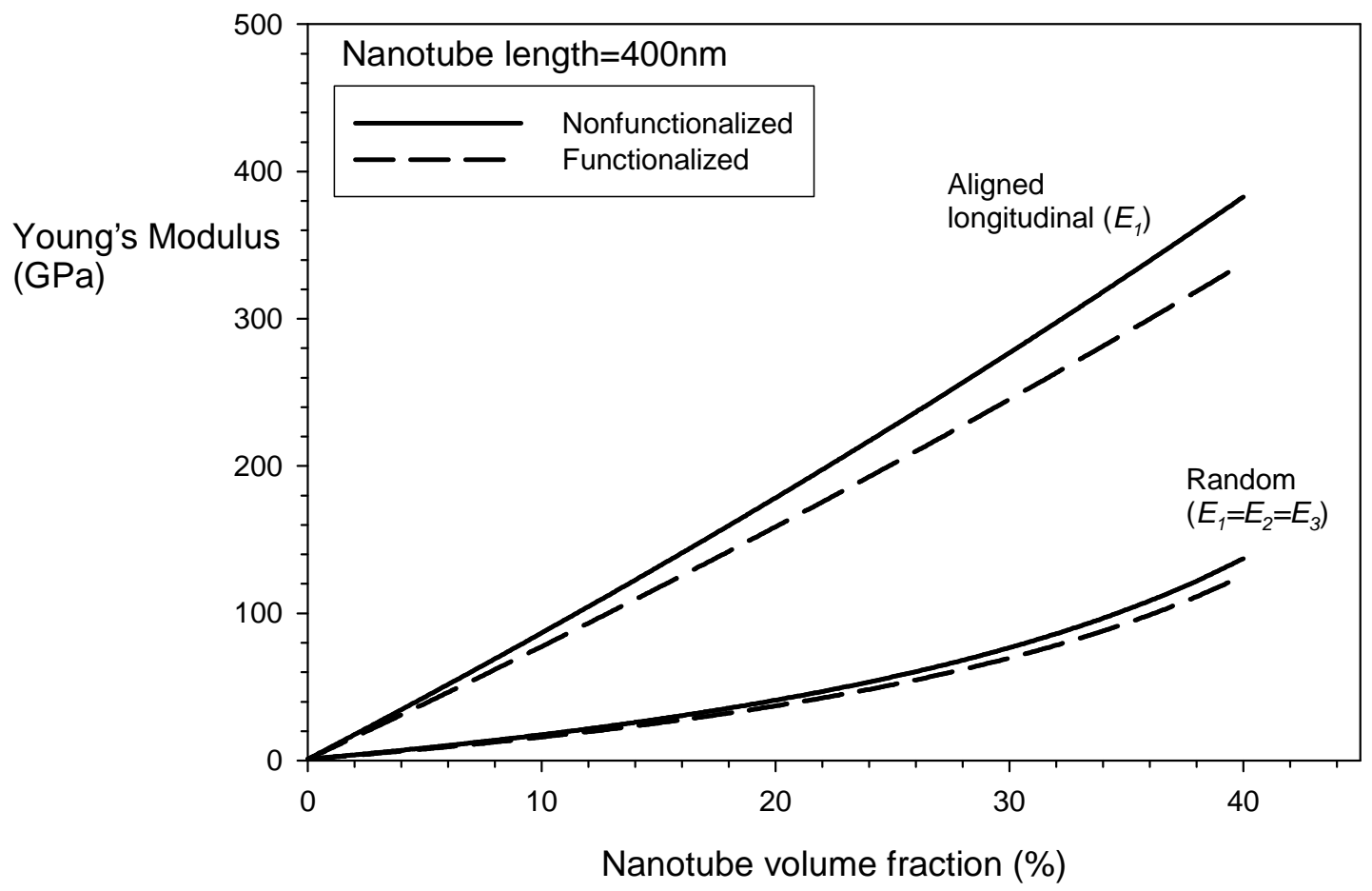

Figure 4: Effect of nanotube volume fraction on Young's modulus, longitudinal, for fixed nanotube length.

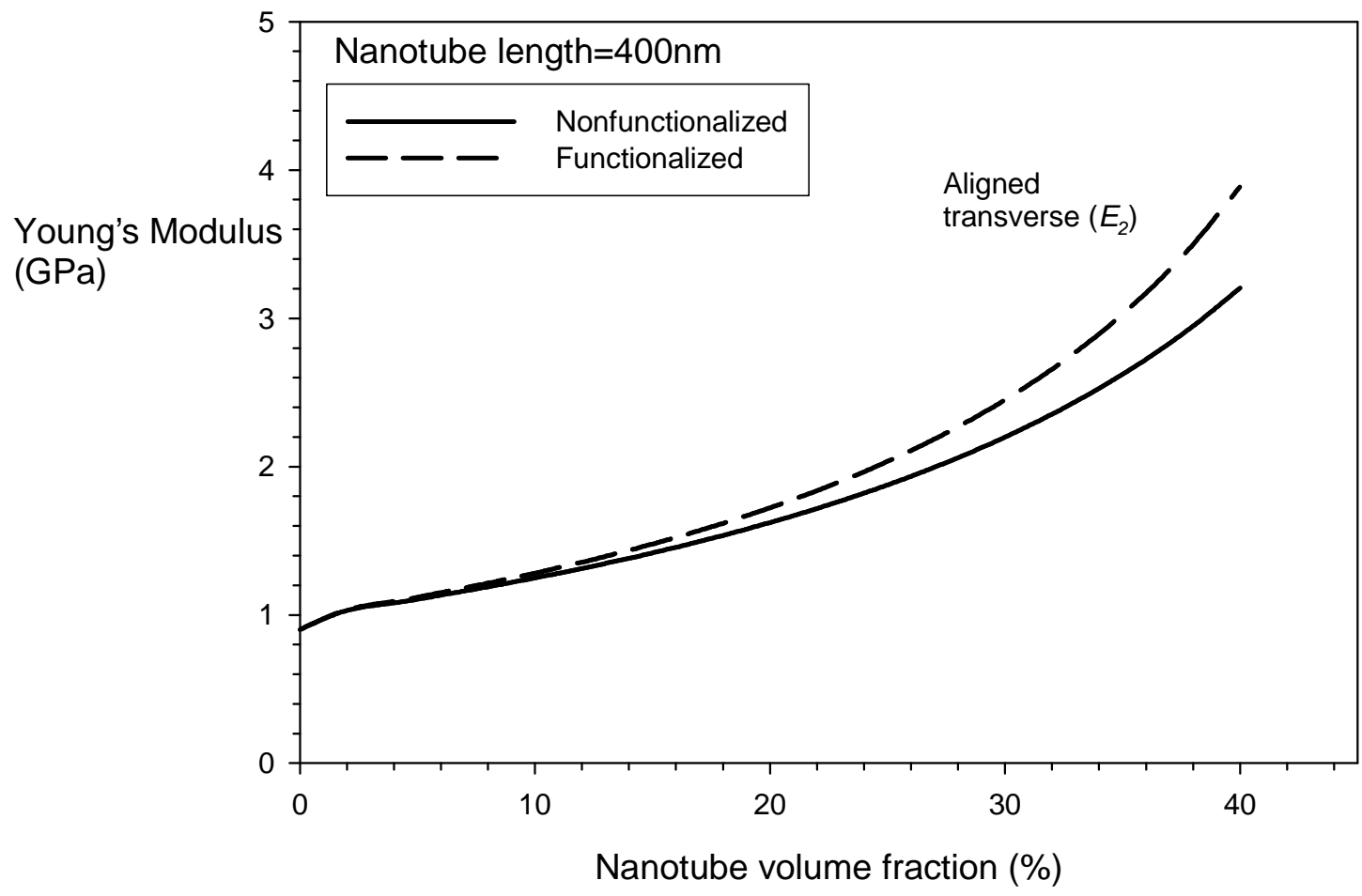

Figure 5: Effect of nanotube volume fraction on Young's modulus, transverse, for fixed nanotube length. 


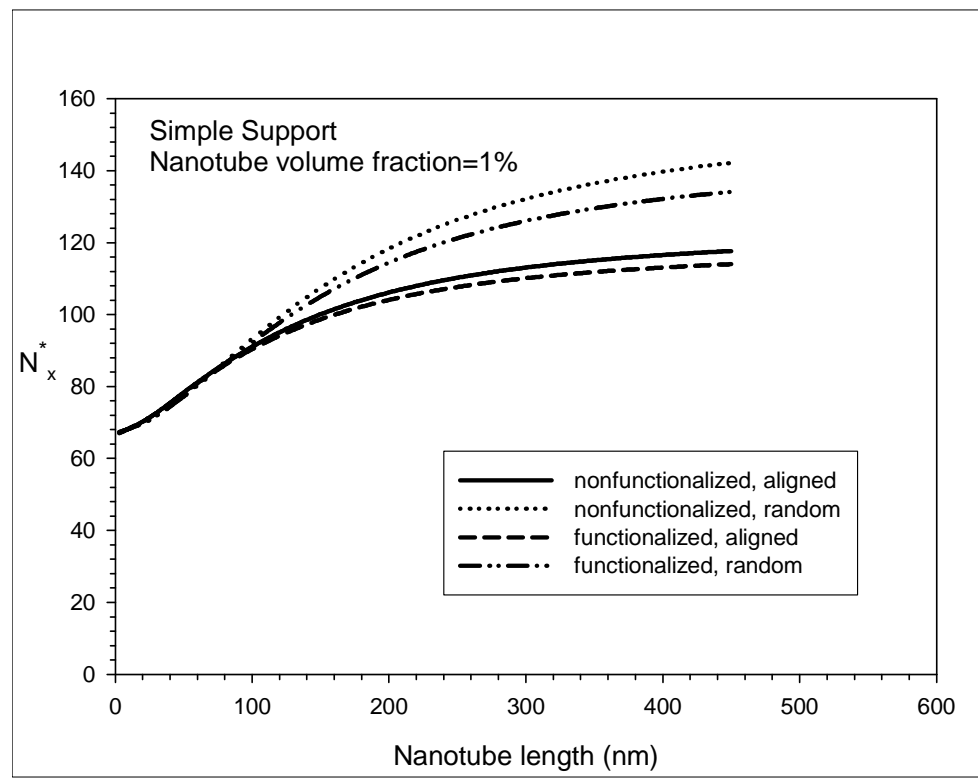

Figure 6: Normalized buckling load for compression, simple support, fixed volume fraction.

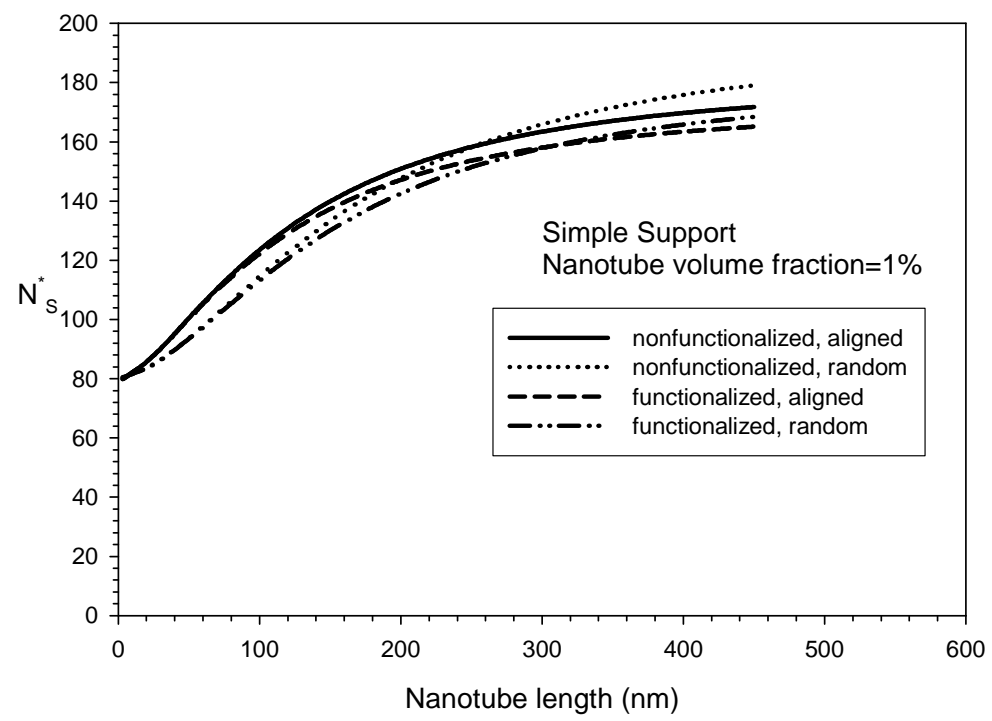

Figure 7: Normalized buckling load for shear, simple support, fixed volume fraction.

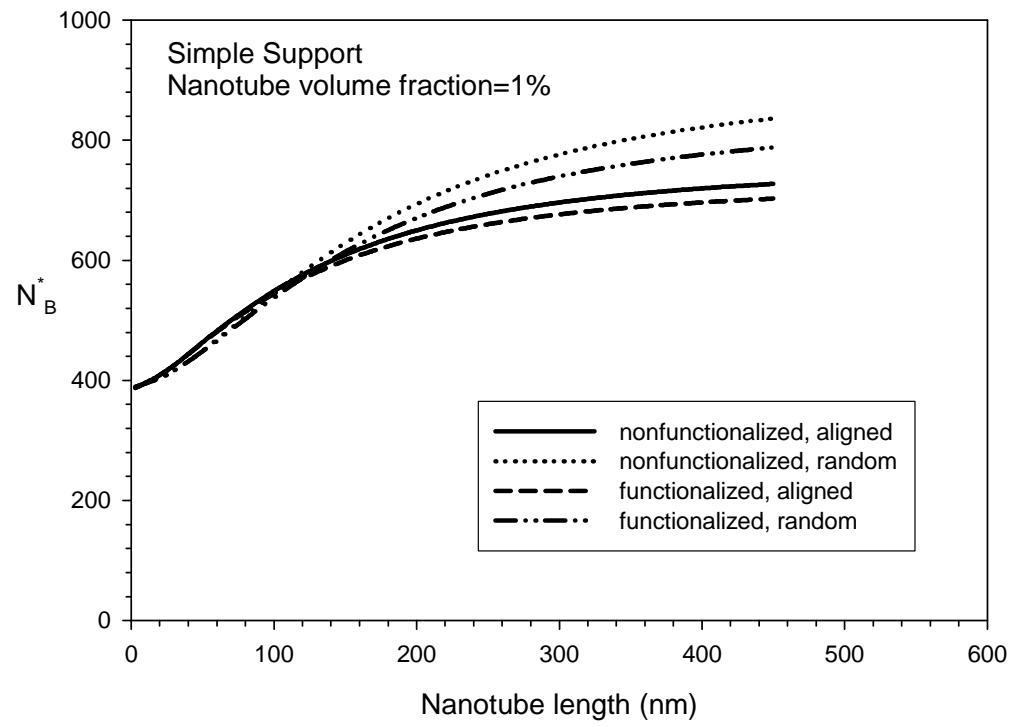

Figure 8: Normalized buckling load for bending, simple support, fixed volume fraction. 


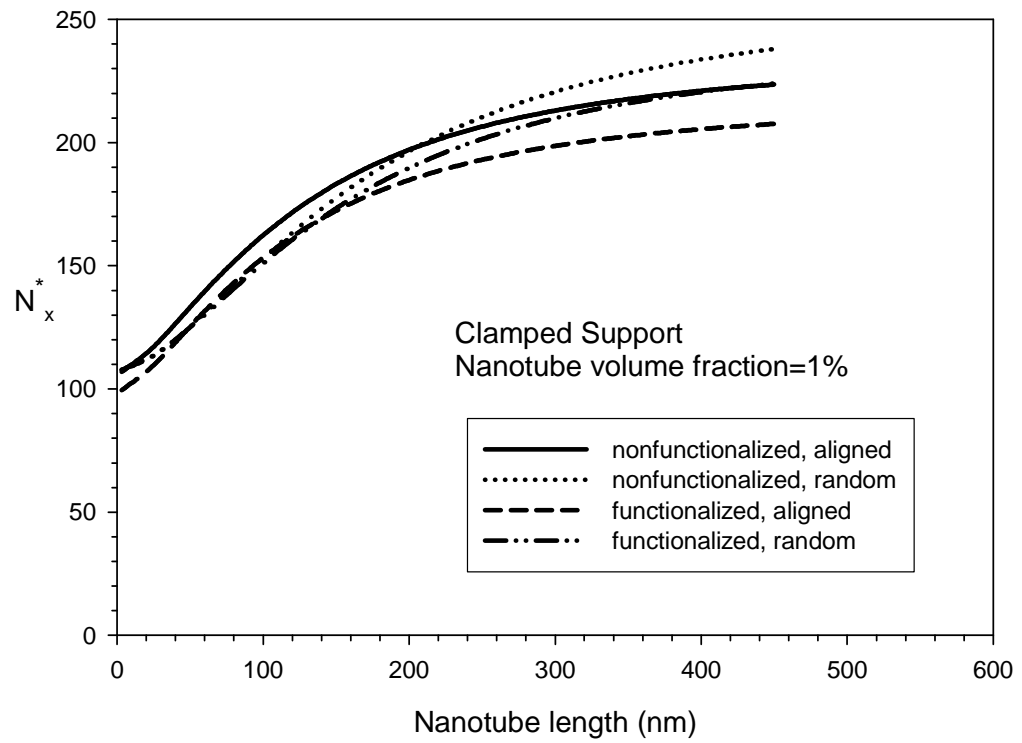

Figure 9: Normalized buckling load for compression, clamped support, fixed volume fraction.

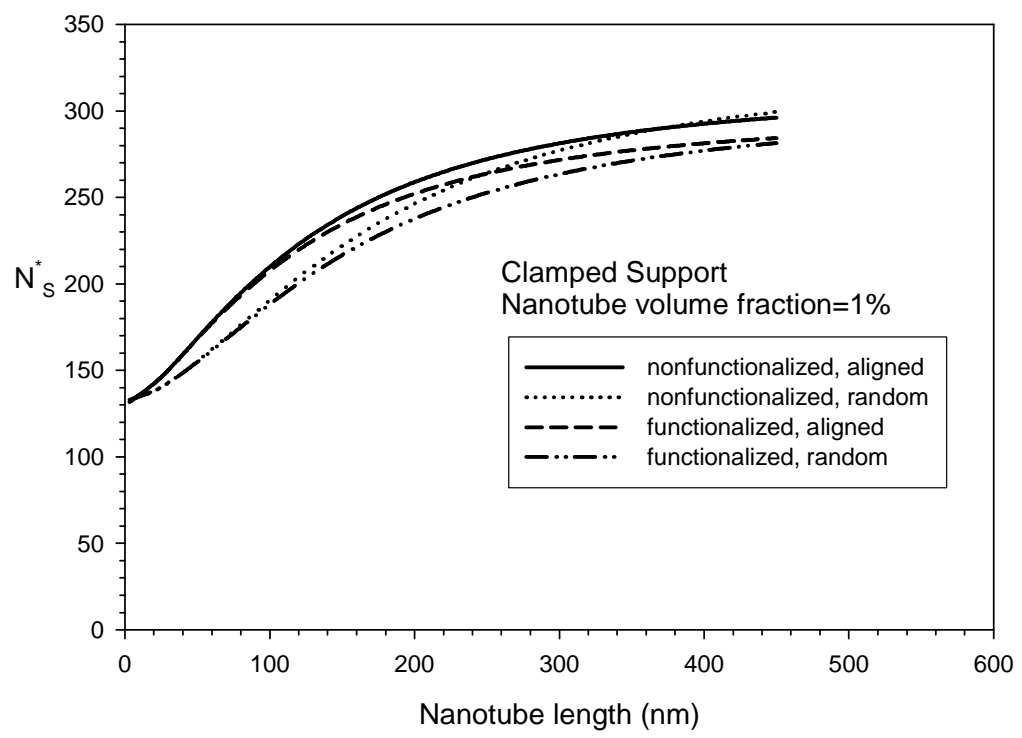

Figure 10: Normalized buckling load for shear, clamped support, fixed volume fraction.

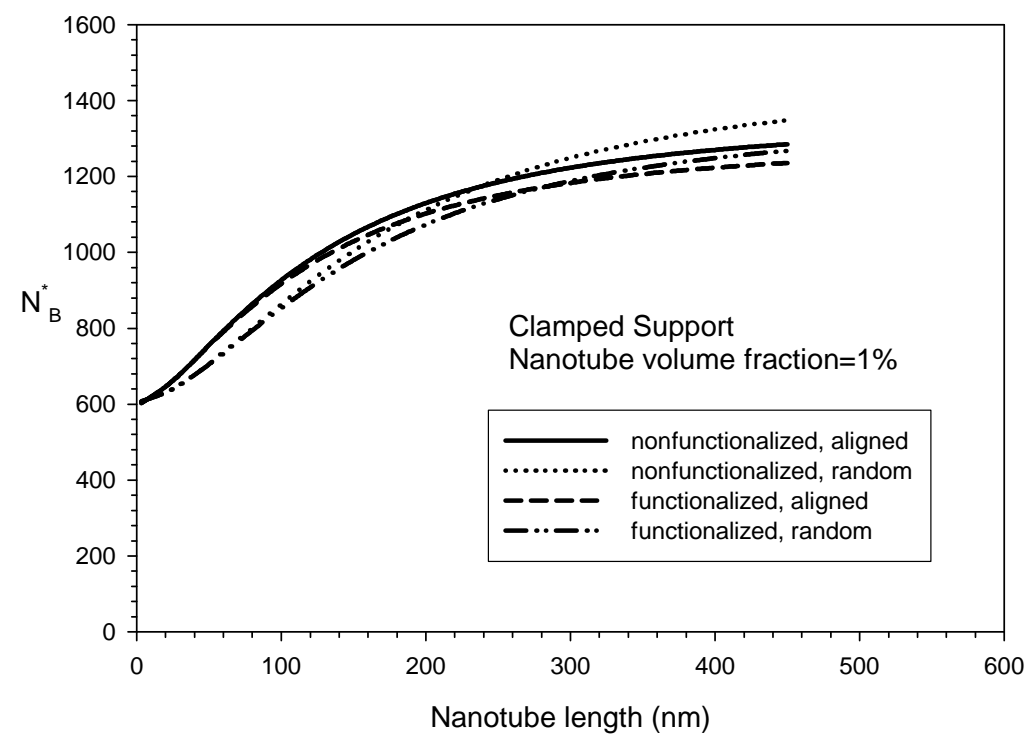

Figure 11: Normalized buckling load for bending, clamped support, fixed volume fraction. 


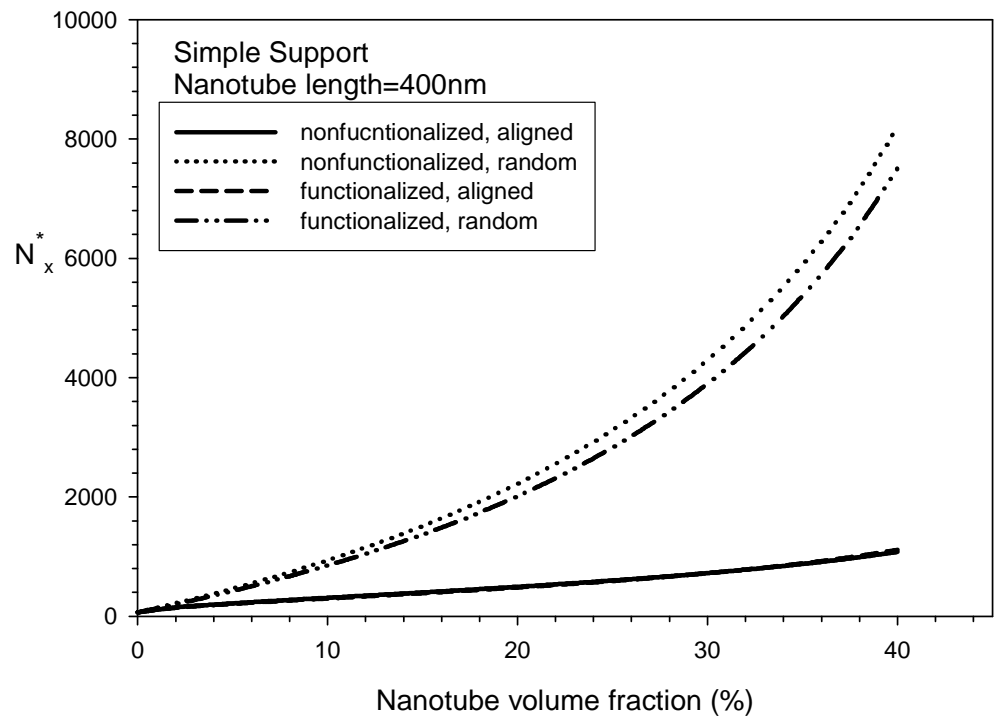

Figure 12: Normalized buckling load for compression, simple support, fixed nanotube length.

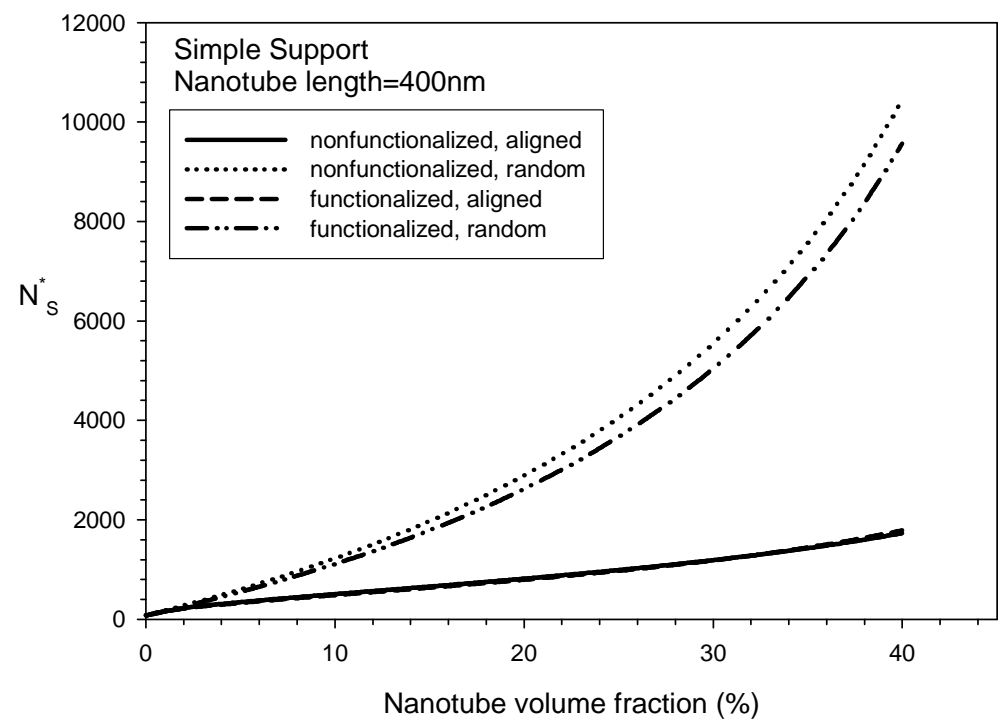

Figure 13: Normalized buckling load for shear, simple support, fixed nanotube length.

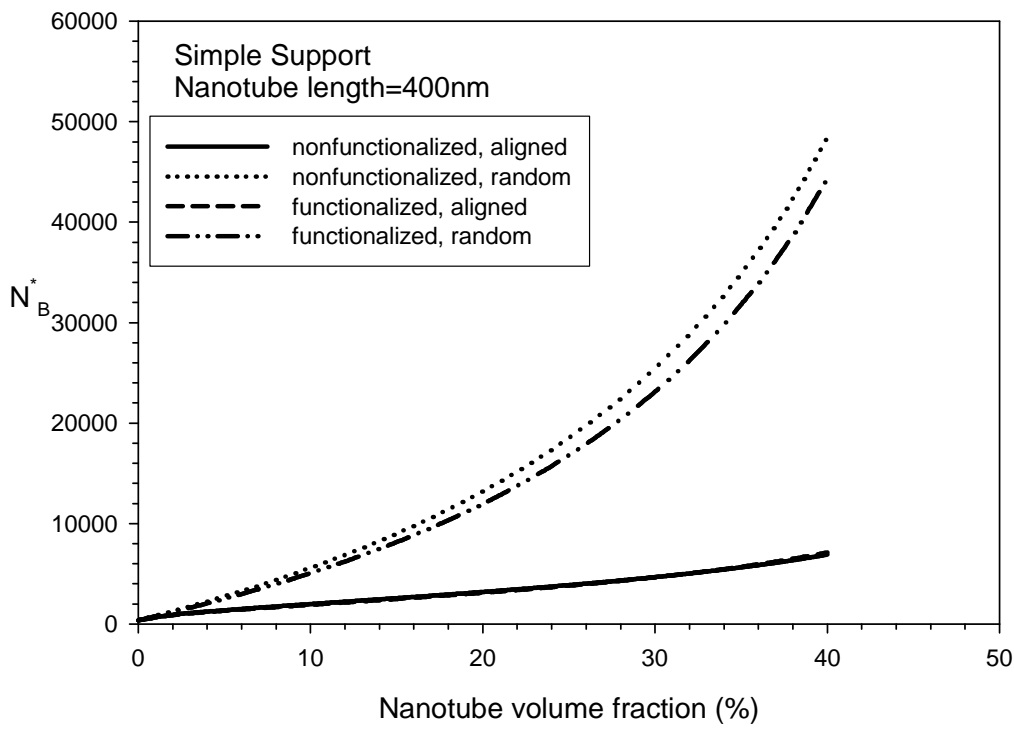

Figure 14: Normalized buckling load for bending, simple support, fixed nanotube length. 


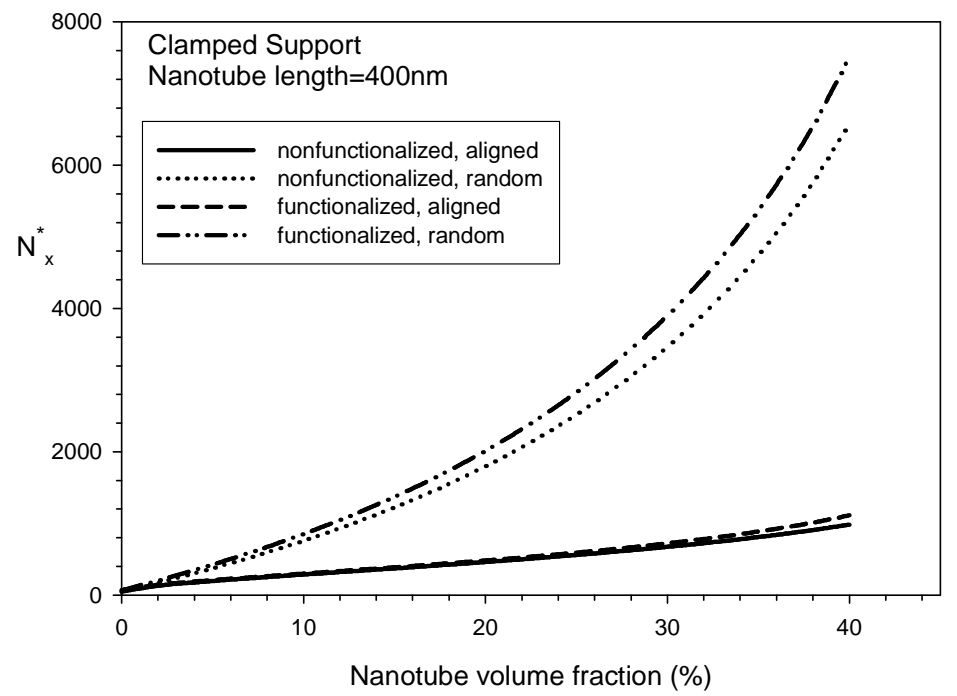

Figure 15: Normalized buckling load for compression, clamped support, fixed nanotube length.

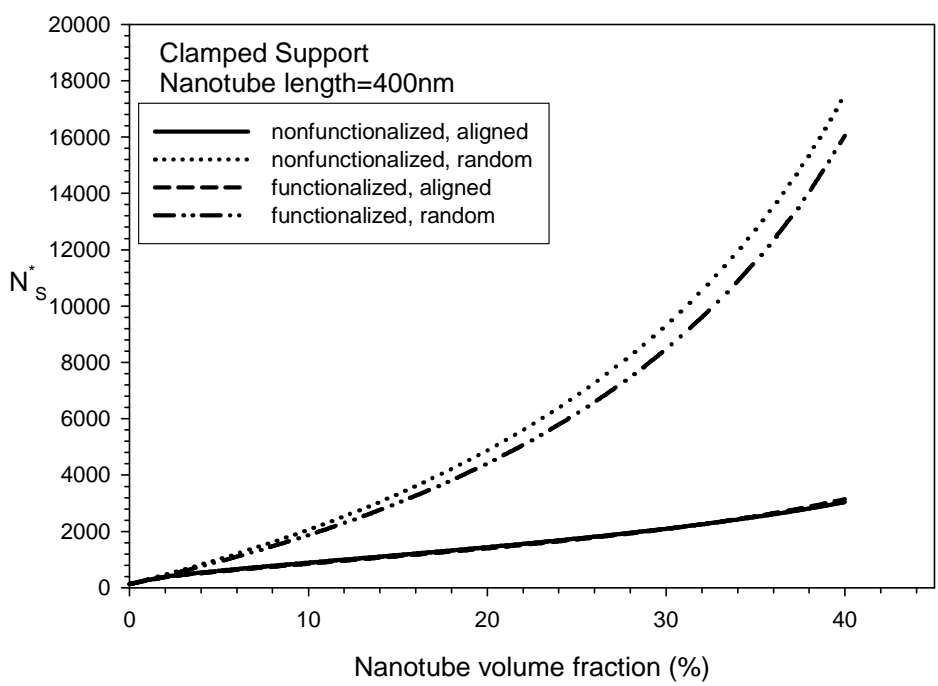

Figure 16: Normalized buckling load for shear, clamped support, fixed nanotube length.

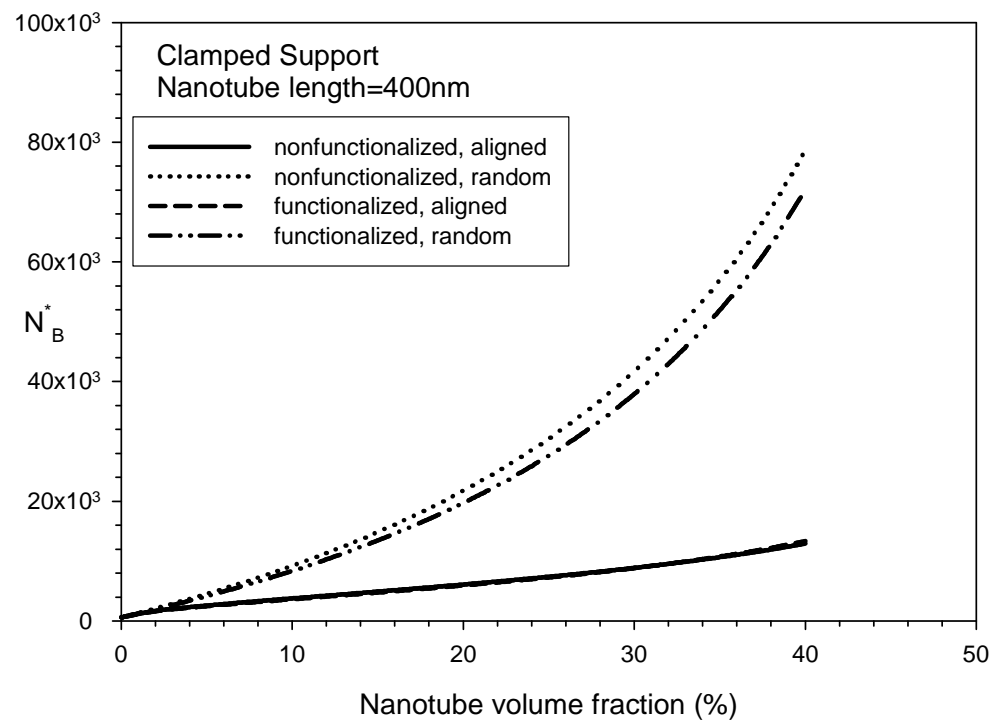

Figure 17: Normalized buckling load for bending, clamped support, fixed nanotube length. 